\title{
An Audit Investigating the Usefulness of a Hospital-Based 4-Week First Postoperative Review Following Routine Cataract Surgery
}

\author{
Louise F. Porter ${ }^{1^{\star}}$, Amy Smith ${ }^{2}$, Aaron D. Showman ${ }^{3}$ \\ and S. Ahmed Sadiq ${ }^{1}$ \\ ${ }^{1}$ University of Manchester, Manchester Academic Health Sciences Centre, \\ Manchester Royal Eye Hospital, Oxford Road, Manchester, United Kingdom. \\ ${ }^{2}$ Royal Berkshire NHS Foundation Trust, London Road, Reading, United Kingdom. \\ ${ }^{3}$ Aston University, School of Life and Health Sciences, Optometry Department, Birmingham,
} United Kingdom.

Short Communication
Received $4^{\text {th }}$ August 2011 Accepted $11^{\text {th }}$ November 2011 Online Ready $24^{\text {th }}$ November 2011

\section{ABSTRACT}

Aim: Timing of postoperative review after routine phacoemulsification cataract surgery in the English National Health Service is not specified in the Royal College of Ophthalmologists Cataract Surgery Guidelines. There are wide variations in when the first postoperative review occurs in routine practice. This audit assesses the usefulness of a 4-week first postoperative review in the hospital setting, in the context of high volume routine cataract surgery.

Setting: Manchester Royal Eye Hospital cataract treatment centre based at Withington Hospital, UK.

Methods: Retrospective review of 200 consecutive sets of case notes of patients undergoing routine phacoemulsification cataract surgery with standard 4-week postoperative review using a proforma.

Results: $88 \%$ of patients were discharged at the 4 -week review for this episode. $4.5 \%$ of patients required earlier unplanned review. $16.5 \%$ of patients experienced a postoperative problem, anterior uveitis was the commonest complication. $15 \%$ of patients were referred from the postoperative clinic for investigation or continuing management of co-existing ocular disease. 
Conclusions: A four-week hospital-based first post-operative review following routine cataract surgery does not serve the purpose of treating sight-threatening complications. A substantial proportion of routine cataract surgery patients (88\%) were discharged at this review. A routine 4-week follow-up review may therefore not be required within a hospital setting in many cases. It would be a reasonably safe option to consider early discharge of such patients to an accredited community optometrist for routine postoperative review and refraction in the presence of careful patient selection and good access to emergency eye care facilities.

Keywords: Cataract surgery; review; complications; community discharge.

\section{INTRODUCTION}

Phacoemulsification cataract surgery with intra-ocular lens implantation (IOL) is the most common elective surgical intervention (Department of Health, 2008). With an increasingly aging population and improvements in surgical outcomes, the demand for cataract surgery has risen significantly (Thirumalai et al., 2003; Fedorowicz et al., 2011). In the English National Health Service (NHS) health care delivery is changing, and resources are limited. Following "Action on Cataracts" and other initiatives (Department of Health, 2004) cataract management has become a multi-professional, high volume process.

Payment by results and ever-reducing times to outpatient appointments and surgery are encouraging departments to seek different ways of delivering their service. Importantly, there are no specific guidelines on follow-up of patients after uncomplicated phacoemulsification cataract surgery in the English NHS. The Royal College of Ophthalmologists Cataract Surgery Guidelines, September 2010, suggest discharge on the day of surgery by an appropriately trained member of staff, with postoperative written instructions, medications, appointments and emergency contact details given to the patient, with a final review at a later date (Royal College of Ophthalmologists, 2010). The timing of the final review date is not specified and consequently there are wide variations (from one to 28 days) in when it occurs in routine practice (Thirumalai et al., 2003; Tinley et al., 2002; Saeed et al., 2007). First day review is no longer in widespread use in uncomplicated cases and there is evidence to indicate that this first postoperative review can be safely deferred until 2 weeks in routine cases, if adequate patient counselling is given, and there is provision of good access to emergency eye services (Thirumalai et al., 2003; Tinley et al., 2002; Zamvar and Dhillon, 2005; Saeed et al., 2007; Alwitry et al., 2006).

Manchester Royal Eye Hospital has a high volume cataract treatment centre (CTC) based in a satellite unit at Withington Hospital, UK. Routine postoperative cataract patients at the CTC are reviewed 4-weeks postoperatively. This review is performed either by an ophthalmologist or an appropriately trained optometrist. No other routine ophthalmic review is organised for patients selected for this pathway. It is expected that most patients will be discharged at the 4-week visit, unless they are due to undergo cataract surgery on the other eye. According to our local statistics, omitting this 4-week follow-up review at the hospital would reduce the number of review slots required by 4000 per year, (personal communication). This audit assesses the necessity of this 4-week hospital postoperative review in the context of high volume cataract day-case surgery by examining outcomes of the 4-week postoperative review. With increasing pressures on our cataract surgery services 
in the United Kingdom, results from our research study may support evidence-based decisions to plan economically viable strategies to aid efficiency of the hospital eye services.

\section{EXPERIMENTAL DETAILS}

A review of 200 consecutive sets of case notes, equivalent to the volume of two weeks of surgery, of patients undergoing routine phacoemulsification cataract surgery with a standard 4-week postoperative first review was performed. There were no planned earlier reviews and patients with planned earlier reviews, for whatever reason, were excluded from this series. All consecutive patients underwent surgery between $2^{\text {nd }}$ January 2008 and $29^{\text {th }}$ January 2008 and were identified from an electronic register. Case notes were reviewed by a single cataract surgeon (LFP) using a proforma requesting details of surgery, co-morbidities, medications, emergency reviews and any unanticipated problems. Data collection occurred between the $5^{\text {th }}$ February 2009 and $31^{\text {st }}$ March 2009.

All procedures were performed using either topical anaesthesia or sub-tenon's anaesthesia. Standard postoperative treatment following routine cataract surgery was $\mathrm{g}$. Maxitrol qds for 4-weeks (dexamethasone, neomycin, polymyxin B sulphate), or g. Betnesol-N (betamethasone and neomycin) qds for 4 weeks. Patients at clinical risk from an intra-ocular pressure (IOP) spike were given one or two doses of oral acetozolamide, at the discretion of the operating surgeon. Patients were discharged on the day of surgery by an appropriately trained member of staff with postoperative written instructions, medications, appointments and emergency contact details, as per Royal College of Ophthalmologists best practice recommendations (Royal College of Ophthalmologists, 2010).

Postoperative review was performed 4 -weeks after the procedure at the hospital by an optometrist or ophthalmologist and whether planned or unplanned included measurement of visual acuity (VA) (uncorrected and pin-hole assisted), IOP measurement, anterior segment examination by slit-lamp biomicroscopy, and assessment of wound integrity by fluorescein testing. When the VA was less than $6 / 12$, a dilated posterior segment examination was also carried out.

\section{RESULTS}

126 patients $(63 \%)$ were reviewed by an optometrist, $74(37 \%)$ by an ophthalmologist (figure 1). 176 patients (88\%) were discharged at the 4-week follow-up review for this particular episode. 24 patients were not discharged. Reasons included postoperative uveitis in 14 cases, suspected cystoid macular oedema (CMO) in 5 cases, and other causes such as corneal abrasion, conjunctivitis and allergy to topical medication in the remaining 5 cases (figure 1).

Demographic details are shown in table 1 and ocular co-morbidities in table 2.

Glaucoma, pseudoexfoliation, age-related macular degeneration (AMD), diabetic retinopathy, amblyopia, previous retinal detachment, vitrectomy, high myopia ( $>8.00$ Dioptres), hypermetropia (>5.00 Dioptres), previous strabismus surgery, previous uveitis and Fuch's endothelial dystrophy were included as co-morbid factors, because they may all influence the risk of intraoperative and postoperative complications (Henderson, 2007). Oral alpha-blockers may also increase the risk of intraoperative complications and 13 patients were taking these agents (Henderson, 2007). 


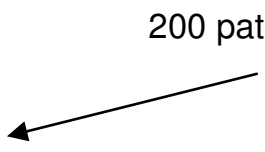

126 patients $(63 \%)$ reviewed by optometrist
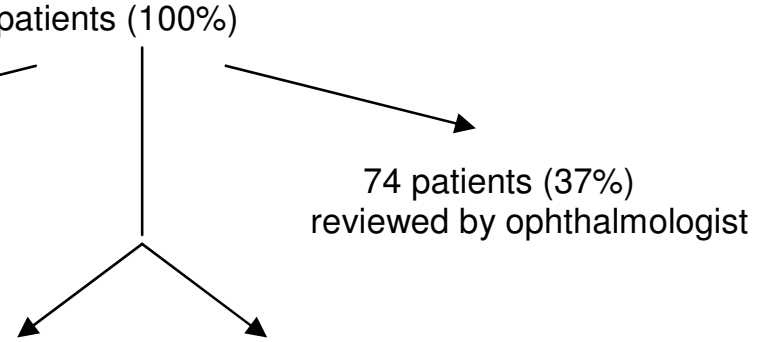

176 patients (88\%) 24 patients (12\%)

Discharged Not discharged for this episode

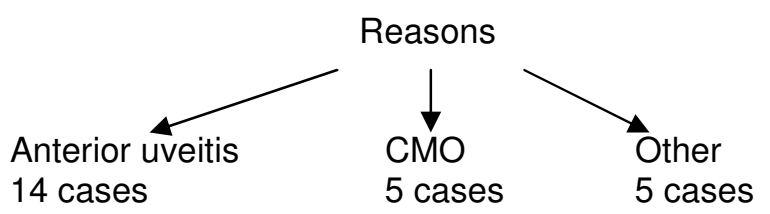

Figure 1: Flowcharts of outcomes for 200 routine cataract surgery patients (CMO: cystoid macular oedema).

Table 1: Age distribution of patients undergoing cataract surgery

\begin{tabular}{lc}
\hline $\begin{array}{l}\text { Age groups of patients } \\
\text { undergoing cataract surgery }\end{array}$ & Percentage \\
\hline$<60$ & $9 \%$ \\
$60-70$ & $24 \%$ \\
$71-80$ & $37 \%$ \\
$>80$ & $30 \%$ \\
\hline
\end{tabular}

Table 2: Proportion of patients with ocular co-morbidities

\begin{tabular}{lc}
\hline Ocular co-morbidities & Percentage \\
\hline None & $45 \%$ \\
One ocular co-morbidities & $45 \%$ \\
Two ocular co-morbidities & $9 \%$ \\
$>$ Two ocular co-morbidities & $1 \%$ \\
\hline
\end{tabular}

Surgery was uncomplicated in $93 \%$ of cases. $7 \%$ of patients (14 patients) suffered a minor intraoperative complication. Floppy iris syndrome was the most common complication (6 patients); other problems included conjunctival chemosis (4 patients), intraoperative miosis (1 patient), anterior capsular tear (1 patient), corneal abrasion (1 patient) and IOL insertion problem (1 patient). None of these minor issues were deemed to require an earlier review.

28 patients (14\%) experienced a postoperative problem detected at the routine 4-week follow-up appointment by the optometrist or ophthalmologist (figure 2). 


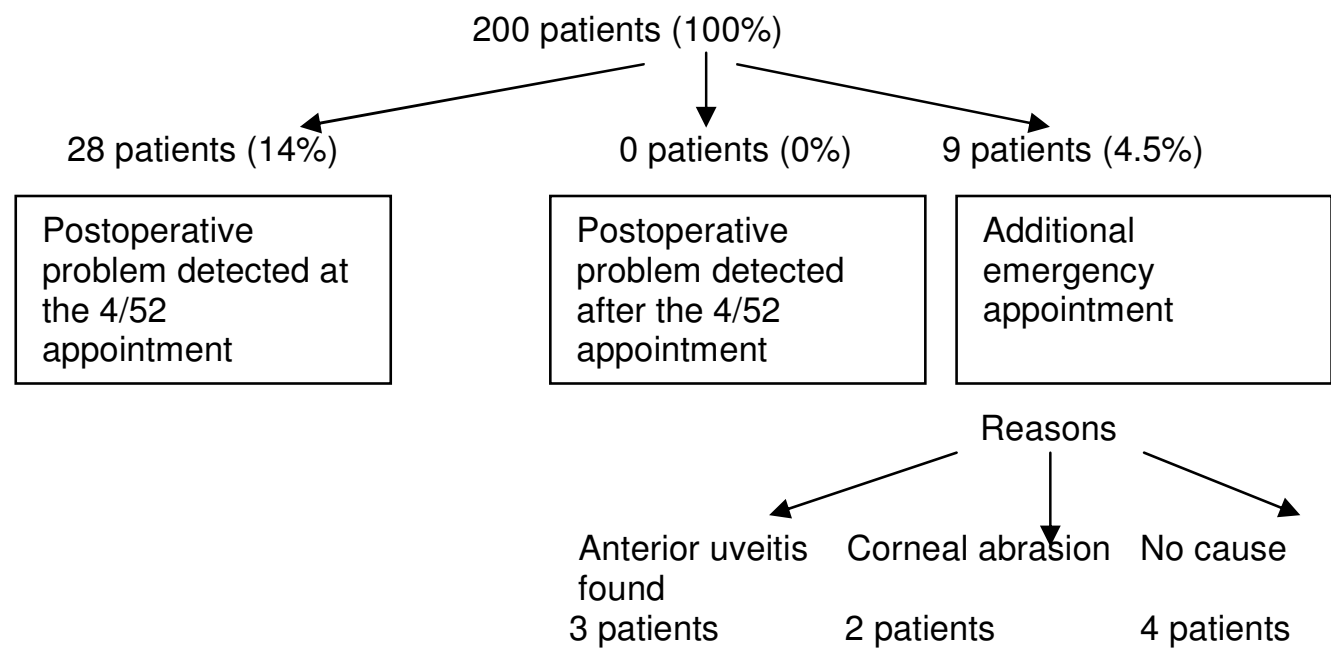

Figure 2: Flowchart of postoperative problems for 200 cataract surgery patients

Anterior uveitis, occurring in 14 cases, was the commonest postoperative problem. 6 of these cases were very mild, requiring only a prescription for topical steroids once daily beyond the 4-week period, however one patient with raised IOP in association with postoperative uveitis was diagnosed at the 4-week postoperative review. Other reported complications included $\mathrm{CMO}$ in 5 patients, corneal abrasion (2 patients), conjunctival injection ( 3 patients), posterior capsular opacification (2 patients) and allergy to topical medication (2 patients). Of note, only 9 patients $(4.5 \%)$ required unplanned review prior to the 4-week routine follow-up appointment. The reasons for early review were anterior uveitis (3 patients), presenting in the second week following the surgery, and corneal abrasion (2 patients), presenting on the first post-operative day in both cases. 4 patients attended with concerns regarding ocular discomfort or reduced vision within the first week of surgery but no abnormalities were found in these patients. One patient also received advice over the telephone.

31 patients (15\%) were referred from the postoperative review clinic for investigation or management of co-existing ocular disease. The pathologies concerned were predominantly diabetic retinopathy (8 patients) and glaucoma-related pathologies (14 patients). 2 patients were referred for YAG laser capsulotomy. The remaining 7 patients were referred for other reasons such as ARMD, branch retinal artery occlusion, and iris naevus.

\section{DISCUSSION}

It is difficult with the current pressures on our cataract surgery services in England to plan an economically viable strategy to detect postoperative complications at a pre-symptomatic stage and all cost-reducing approaches will be a compromise (Tinley et al., 2002). Certainly a 4-week first postoperative review in the hospital setting does not appear to serve this purpose, because serious and sight-threatening complications tend to occur early in the postoperative period (raised IOP, endophthalmitis, anterior uveitis) and earlier hospital review is necessary if these problems are to be totally averted. However Saeed et al. and Alwitry et al. provided the evidence, now in widespread practice, that in the interests of costefficiency first post-operative reviews after routine cataract surgery can be deferred for 
several weeks in most cases (Saeed et al., 2006; Alwitry et al., 2007, Royal College of Ophthalmologists, 2010).

We demonstrate that despite the high proportion of patients with ocular co-morbidities in our series, very few patients (4.5\%) required unplanned review prior to the 4-week follow-up appointment, and there were no serious vision-threatening complications or increased reattendance rates for vision-threatening complications. The total number of complications observed over the time period assessed is in line with regular postoperative hospital audits carried out at a local level by operating cataract surgeons within the Central Manchester Foundation Trust. At the 4-week review appointment there was a high discharge rate (88\%).

The 4-week postoperative review in our centre predominantly serves the purposes of collecting outcome data, reviewing medication, discussing second eye surgery where appropriate, and providing advice on spectacle prescription. In $15 \%$ of cases, predominantly in glaucoma-related pathologies and diabetic retinopathy, it also serves the function of enabling follow-up for co-existing eye disease.

In this audit there was a higher proportion of younger patients and patients with ocular comorbidities than the reported national averages. For example, $33 \%$ of patients in this study were aged under 70, versus 22\% reported in National Cataract Survey (Desai et al., 1999). For the purposes of accuracy this audit recorded a wide range of co-existing pathologies as ocular co-morbidities, helping to explain the higher number of patients with ocular comorbidities in this review compared with the quoted national average of $28 \%$ (Desai et al., 1999). It is difficult therefore to firmly establish how much the patient group in this audit is comparable to the case mix undergoing high volume cataract surgery in other day-case units. However in the context of high numbers of patients with ocular co-morbidities, it is likely that the case mix biases towards cataract surgery cases in which complications are more likely.

The retrospective nature of the study may limit the precision with which observations have been collected. However problems of missing data were minimised by collecting accident and emergency attendance notes for the period under study and ensuring that all patients were questioned at the 4-week review regarding any earlier problems and unplanned clinic attendances. The number of patient notes reviewed also makes the results more robust. Notes were reviewed 14 months after the initial surgery, enabling accurate assessment of any post-operative problems that may have presented after the 4-week review. Interestingly no post-operative problems were detected after this review.

Since $88 \%$ of patients were discharged at the 4-week hospital post-operative review, it appears reasonable to consider direct discharge of such patients to an accredited community optometrist for routine review and refraction, rather than arranging a 4-week postoperative review within a hospital setting for uncomplicated cataract surgery. A fast-track referral service from accredited community optometrists to the ophthalmology-led emergency system could be put in place for the small number of patients with a postoperative problem identified at this review. A fast track system from community optometrists is already in place at the Manchester Royal Eye Hospital for direct referral of patients with urgent ocular problems to the acute ophthalmology services. A separate direct referral system is in place for glaucoma suspects and wet AMD patients using appropriately trained community optometrists and has shown that such a model can work in practice (Henson et al., 2003). 
Omitting the 4-week follow-up review at the hospital would reduce the number of review slots required by approximately 4000 per year according to our local statistics (personal communication) enhancing efficiency of the hospital service. Indeed 4800 surgeries per year are performed at WCH on patients who do not have significant co-morbidities. We have shown that $88 \%$ of patients on the review pathway audited in this report were discharged at the 4-week postoperative review. These results imply that up to 4080 patients per year are simply directly discharged at the time of the 4-week first postoperative review appointment. Another 4000 surgeries are performed at $\mathrm{WCH}$ per year on patients with significant comorbidities that exclude them from the four-week first postoperative review follow-up system audited in this report, these patients are seen at the Manchester Royal Eye Hospital at a time established by their operating surgeon. For this model to work in practice, adequate patient counseling and good access to emergency eye care facilities would remain important. Patients would not have to attend a hospital, with the associated transport problems and waiting times. In addition, careful patient selection would exclude those with glaucoma or diabetic retinopathy and those requiring second eye surgery.

Further research focusing on establishing the best timing for first postoperative review would be beneficial before introducing major changes to the current postoperative protocol. Our audit, nonetheless emphasises that a four-week hospital-based first postoperative review following routine cataract surgery does not serve the purpose of treating sight-threatening complications. It further demonstrates that a substantial proportion of routine cataract surgery patients (88\%) were discharged at this review. This review therefore need not take place in a hospital setting and could be performed by accredited optometrists in a community setting. The benefits of omitting the 4-week follow-up review in a hospital setting are considerable, with a potential for 4000 review slots to be freed up per year (personal communication). In conclusion, results from our audit may support future evidence-based decisions to plan economically viable strategies to detect postoperative complications after cataract surgery, further enhance the role of community optometry and aid efficiency of the hospital eye services.

\section{ACKNOWLEDGEMENT}

NIHR Biomedical Research Centre and Central Manchester and Manchester Children's Foundation NHS Trust, United Kingdom, provided advice for this study. All authors at the time of the study were employed by this organization.

The authors would like to thank Linda Ellis and Sophie Harper for their assistance with this study.

\section{COMPETING INTERESTS}

The authors declare no conflict of interest. No financial support was received for this study.

\section{REFERENCES}

Alwitry, A., Rotchford, A., Gardner, I. (2006) First day review after uncomplicated phacoemulsification: Is it necessary? Eur J. Ophthalmol., 16(4), 554-9.

Desai, P., Reidy, A., Minassian, D. (1999). Profile of patients presenting for cataract surgery in the UK: National data collection. Br J Ophthalmol., 83, 893-896. 
Department of Health National Eye Care Plan, May (2004). http: www.dh.gov.uk/ab/Archive/Eyecareservicesgroup/DH_100100.

Department of health. (2008). Hospital activity and episode statistics. http://www.doh.gov.uk/public/stats1.htm

Fedorowicz, Z., Lawrence, D., Gutierrez, P., van Zuuren, E.J. (2011). Day care versus inpatient surgery for age-related cataract. Cochrane Database Syst Rev., 6(7), CD004242.

Henderson, B. A. (2007). Essentials of cataract surgery. Thorofare NJ: SLACK Incorporated, pp.175-263.

Henson, D. B., Spencer, A. F., Harper, R., and Cadman, E. J. (2003). Community refinement of glaucoma referrals. Eye, 17, 21-26.

Saeed, A., Guerin, M., Khan, I., Keane, P., Stack, J., Hayes, P., Tormey, P., Mulhern, M., Beatty, S. (2007) Deferral of first review after uneventful phacoemulsification cataract surgery until 2 weeks, randomized controlled study. J Cataract and Refract Surg., 33(9), 1591-1596.

Thirumalai, B., Baranyovits, P. (2003). Intraocular pressure changes and its implications on patient review after phacoemulsification. J Cataract Refract Surg., 29, 504-507.

Tinley, C. G., Frost, A., Hakin, K., N., McDermott, W., Ewings, P. (2002). Is visual outcome compromised when next day review is omitted after phacoemulsification surgery? J Cataract and Refract Surg., 28, 977-981.

Royal College of Ophthalmologists Cataract Surgery Guidelines. (2010). London, The Royal College of Ophthalmologists. Available at: http://www.rcophth.ac.uk/docs/publications/CataractSurgeryGuidelinesSeptember2010 .pdf.

Zamvar, U., Dhillon, B. (2005). Postoperative IOP prophylaxis practice following uncomplicated cataract surgery: a UK-wide consultant survey. BMC Ophthalmol., 5, 24.

(c) 2012 Porter et al.; This is an Open Access article distributed under the terms of the Creative Commons Attribution License (http://creativecommons.org/licenses/by/3.0), which permits unrestricted use, distribution, and reproduction in any medium, provided the original work is properly cited. 